\title{
Dynamic response of water droplet coated silicon MEMS resonators
}

\author{
Angel T-H. Lin, Jize Yan, Ashwin A. Seshia \\ Department of Engineering, University of Cambridge, United Kingdom \\ Email: thal2@cam.ac.uk
}

\begin{abstract}
This paper studies the dynamic response of silicon bulk acoustic mode resonators spotted with water droplets of varying volume on the top surface. Three different cases were compared: (i) bare silicon resonators, (ii) parylene $\mathrm{C}$ coated resonators and (iii) hydrophobic self assembled monolayer coated resonators. Experimentally derived variations in quality factor are compared with those obtained analytically for the electrostatically driven square extensional mode resonator. The measured quality factors showed a good agreement with the models.
\end{abstract}

Keywords- bulk acoustic wave resonator; damping; parylene; hydrophobic SAM;

\section{INTRODUCTION}

Silicon MEMS resonators are being explored as a potential alternative to the quartz crystal microbalance for biological sensing wherein operation in contact with aqueous media is often desirable. The operating principle lies in monitoring the shift in resonant frequency and Quality factor when the target molecule to be detected attaches to an appropriately functionalized surface of the resonator. The frequency resolved mass resolution of a resonator depends on the quality factor $(\mathrm{Q})$ such that the larger the $\mathrm{Q}$ the higher the resolution. However, fluid damping of the resonators in liquid can be significant and the $\mathrm{Q}$ is substantially reduced by associated viscous loss.

Fluid damping in MEMS resonators has been studied widely for a long time [1-4]. However, most of the previous studies have focused on flexural mode resonators or structures actuated by lateral comb drives. Viscous damping models have been derived for radial contour mode disk resonators but were not experimentally verified in liquid.

The aim of this study is to estimate the Q factor of a bulk extensional mode resonator from viscous damping and to predict design rules for optimal $\mathrm{Q}$ in liquid. Two resonator surface coatings are utilized to investigate their effect in reducing viscous damping and compared to the case of bare silicon. Water droplets ranging from 1 to $4.5 \mu \mathrm{L}$ are spotted on the centre of the resonator upper surface, and the resonator response is characterized using differential electrostatic driving and piezoresistive sensing.

\section{DEVICE DESCRIPTION AND SURFACE PREPARATION}

The devices tested are $2.2 \mathrm{MHz}$ single crystal silicon square plate resonators operated in the bulk extensional mode, wherein the square plate undergoes an extension and contraction symmetrically on each side of the square. The suspended square is anchored to the substrate via a T-shape connecting stem located on four corners of the square plate. The resonator is fabricated in a commercial SOI foundry process (MEMSCAP), with one lateral electrode on each side of the square plate. An optical micrograph of the fabricated resonator is shown in Fig. 1 and the resonator parameters are summarized in Table 1. The resonator is actuated using differential electrostatic driving, where two complementary AC signals are used for actuation to cancel out the effect of capacitive parasitic feedthrough [5]. The square-extensional mode is excited by biasing the orthogonally aligned actuation electrodes at opposite DC polarities. The mechanical response is sensed by passing a drain current between two opposite corners of the square plate to readout the resulting variation in the electrical resistance of the vibrating structure due to the piezoresistive effect [6].

Three identical resonators are prepared for testing, one is left bare, the second is coated with $300 \mathrm{~nm}$ of parylene $\mathrm{C}$ using the Speciality Coating Systems PDS 2010 Labcoter, and the $3^{\text {rd }}$ is coated with hydrophobic SAM $(1 \mathrm{H}, 1 \mathrm{H}, 2 \mathrm{H}, 2 \mathrm{H}-$ Perfluorodecyltrichlorosilane, Alfa Aesar) using vapour deposition in vacuum overnight. The water droplets are spotted on the centre of the resonator using micropipettes. The droplets were left to dry in air between successive measurements. The evaporation time for a $1 \mu \mathrm{L}$ water droplet to dry out completely is more than 10 minutes for all three surface coatings, and the measurement time is around 50s, ensuring that for droplet volumes greater than $1 \mu \mathrm{L}$, the water droplet volume does not change by more than $10 \%$ during the measurements.

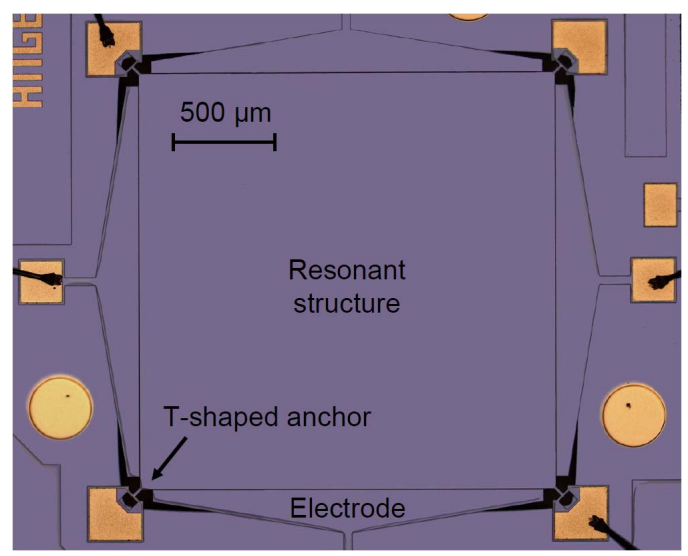

Figure 1: Optical micrograph of the resonator 
Table 1: Resonator parameters

\begin{tabular}{|l|c|c|}
\hline \multicolumn{1}{|c|}{ Parameters } & Symbol & Value \\
\hline Side length & $L$ & $2000 \mu \mathrm{m}$ \\
\hline Thickness & $H$ & $25 \mu \mathrm{m}$ \\
\hline Transduction gap & $g_{0}$ & $2.5 \mu \mathrm{m}$ \\
\hline Length of one electrode & $L_{e}$ & $1880 \mu \mathrm{m}$ \\
\hline Resonant frequency & $f$ & $2.2 \mathrm{MHz}$ \\
\hline $\begin{array}{l}\text { Measured quality factor in air for } \\
\text { bare silicon resonator }\end{array}$ & $Q$ & 11013 \\
\hline
\end{tabular}

\section{ANALYTICAL MODELLING}

Damping in mechanical resonators can arise from a number of sources, including squeeze film damping, viscous drag, acoustic radiation loss via attachments (anchor loss) and other internal loss mechanisms. The quality factor is the ratio of energy stored per cycle to the energy dissipated per radian. For a mechanical resonator, it is given by the expression:

$$
Q=\frac{m \omega_{0}}{b}
$$

where $m, \omega_{0}, b$ is the equivalent mass, angular resonant frequency of the resonator, and damping constant respectively. Since energy loss from all sources adds directly to the total energy loss, the overall Q factor is given by:

$$
\frac{1}{Q_{\text {total }}}=\frac{1}{Q_{\text {vis_drag }}}+\frac{1}{Q_{\text {squeez__film }}}+\frac{1}{Q_{\text {anchor }}}+\frac{1}{Q_{\text {other }}}
$$

\section{A. Viscous damping of laterally vibrating plate}

The viscous damping model developed here for square extensional mode resonators is calculated from the solution of Navier-Stokes equation for an incompressible fluid for oscillatory motion parallel to the surface of the resonator. In the model we follow the procedure as described by Vignola et al [4], and make the fluid contact area $A$ a parameter. If we take the resonator surface as the $y z$-plane, and the fluid region as $x>0$, as shown in figure 2 , the viscous force per unit area is given by [7]:

$$
F_{v i s}=\sqrt{\frac{1}{2} \omega \eta \rho_{\text {fluid }}}(j-1) v
$$

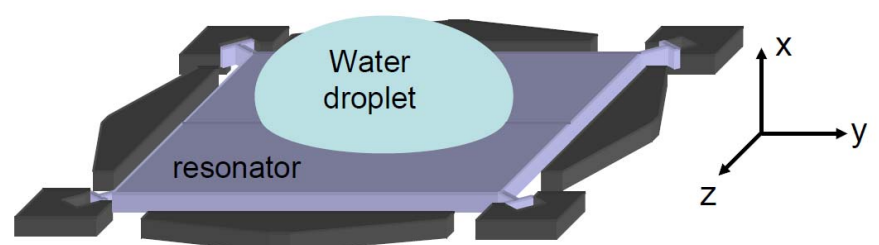

Figure 2: Digram illustrating the water droplet spotted on the centre of the resonator, where the resonator surface is the $y z$-plane, with the fluid region at $x$ $>0$.

where $\eta, \rho_{\text {fluid }}, v$ is the viscosity, density and velocity of the fluid. At $x=0, v=u$, where $u$ is the velocity of the resonator surface, defined by its mode shape equation. If we divide the resonator surface into $N$ equal elements of area $A_{i}$, small enough that the velocity is approximately uniform over $A_{i}$, the energy dissipated can be calculated from the real part of $F_{v i s}$ and the damping constant $b_{v i s, i}$ due to viscous drag at $A_{i}$ is given by:

$$
b_{v i s, i}=\frac{F_{v i s, i} A_{i}}{u_{i}}=\sqrt{\frac{1}{2} \omega \eta \rho_{\text {fuid }}} A_{i}
$$

Assuming that the velocity is uniform in the area element $A_{i}$ and summing up the damping contribution of all such elements, the $\mathrm{Q}$ factor for the resonator due to viscous damping for fluid contact area $A$ is given by:

$$
Q_{\text {viscous }}=\frac{m \omega_{0}}{b_{\text {vis }}}=\frac{\rho_{S i} H L^{2} \omega_{0}}{\sqrt{\frac{1}{2} \omega_{0} \eta \rho_{\text {fluid }}}}=\rho_{S i} H \sqrt{\frac{2 \omega_{0}}{\eta \rho_{\text {fluid }}}}\left(\frac{L^{2}}{A}\right)
$$

where $\rho_{S i}, H, L$ is the density, thickness and length of the square plate resonator. Substituting the water droplet contact area into $A$ for $Q_{\text {water_drop }}$ due to viscous damping from water droplet, and taking the rest of the area covered by air for $Q_{\text {remain_air }}$ due to viscous damping from the air contact, the total $\mathrm{Q}$ due to in-plane viscous drag can be approximated as:

$$
\frac{1}{Q_{\text {vis_drag }}}=\frac{1}{Q_{\text {water_drop }}}+\frac{1}{Q_{\text {remain_air }}}
$$

\section{B. Squeeze film damping}

Squeeze film damping for electrostatically driven resonators can occur between the resonator side walls and the actuation electrodes. The resonator has four electrodes for actuation on each side of the square plate. The damping constant for squeeze film damping for electrode plates of length $L_{e}$, width $H$ and gap size $g_{0}$, is given by [1]:

$$
b_{\text {squeze } f \text { film }}=\frac{96 \eta\left(4 L_{e}\right) H^{3}}{\pi^{4} g_{0}^{3}}=\frac{384 \eta L_{e} H^{3}}{\pi^{4} g_{0}^{3}}
$$

The quality factor due to squeeze film damping is therefore given by:

$$
Q_{\text {squeze_film }}=\frac{m \omega}{b_{\text {squeeze_film }}}=\frac{\rho_{s i} L^{2} \omega_{0} \pi^{4} g_{0}^{3}}{384 \eta L_{e} H^{2}}
$$

The total Q due to fluid damping is given by

$$
\frac{1}{Q_{\text {fluid }}}=\frac{1}{Q_{\text {vis_drag }}}+\frac{1}{Q_{\text {squeeze_film }}}
$$

Figure 3 shows the estimated Q-factor of the square extensional mode resonator due to fluid damping. It can be seen that for ratios of water contact area to resonator top surface area of $2 \%$ or more, the viscous drag from water is the dominant source of energy dissipation. 


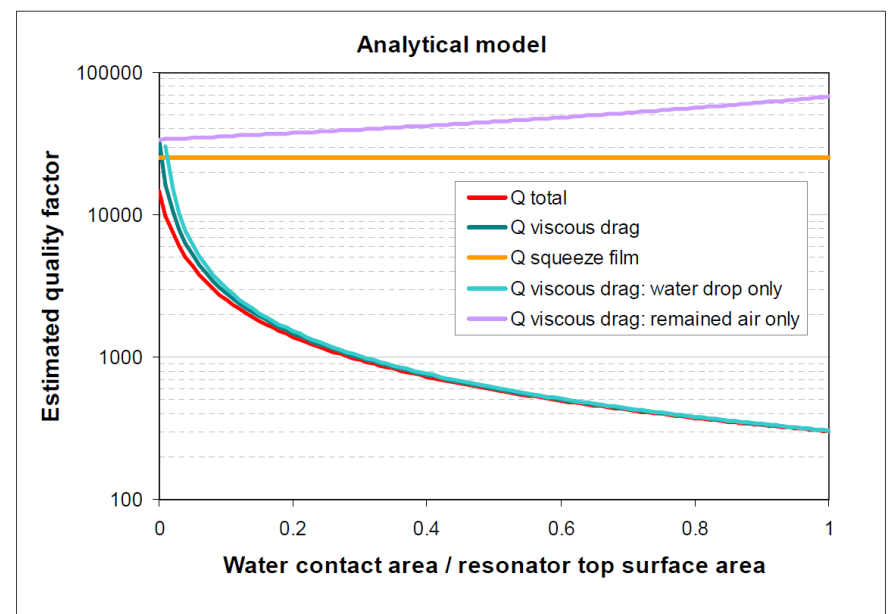

Figure 3: Analytical model for fluid damping estimation, showing in-plane viscous damping from the water droplet is dominant for a water contact area greater than $2 \%$ of the resonator top surface area.

\section{RESULTS AND DISCUSSIONS}

The frequency response plots of the resonators to varying water droplet size are shown in figure 4 . The extracted quality factor and resonant frequency for the measured data is summarized in table 2. Larger droplet volumes could be applied to the SAM and parylene coatings due to higher water contact angle. The largest droplet volume used for each coating is the largest volume in steps of $0.5 \mu \mathrm{L}$ that can be applied before the water flows into the transduction gaps. The smallest droplet volume that can be spotted on the SAM coated resonator is $1 \mu \mathrm{L}$, this is due to the high water contact angle of the hydrophobic surface $\left(\sim 115^{\circ}\right)$.

For all three resonators, there is a decreasing trend in resonant frequency and Q-factor with increasing water droplet volume. For the same droplet volume, the SAM coated resonator showed the highest $\mathrm{Q}$, followed by parylene coating, and the bare silicon resonator had the lowest $\mathrm{Q}$, which indicates that hydrophobic coatings effectively enhanced the performance in a liquid environment due to the reduction in contact area between the water drop and the resonator surface. However, for the similar fluid contact areas (taking the largest droplet volume), the Qs were similar for all three coatings, indicating surface coatings may not have an effect on viscous damping if the effective area of contact with the water droplet is the same.

Figure 5 shows the comparison between estimated Q vs. water contact area and the measured Q vs. water droplet volume. The maximum droplet volume that can be applied is assumed to be the droplet volume that has a water contact area of approximately $\pi L^{2} / 4$ (i.e. $78 \%$ of the top surface area). The trend of decreasing $\mathrm{Q}$ for all three resonators is the same as that given by the analytical model.
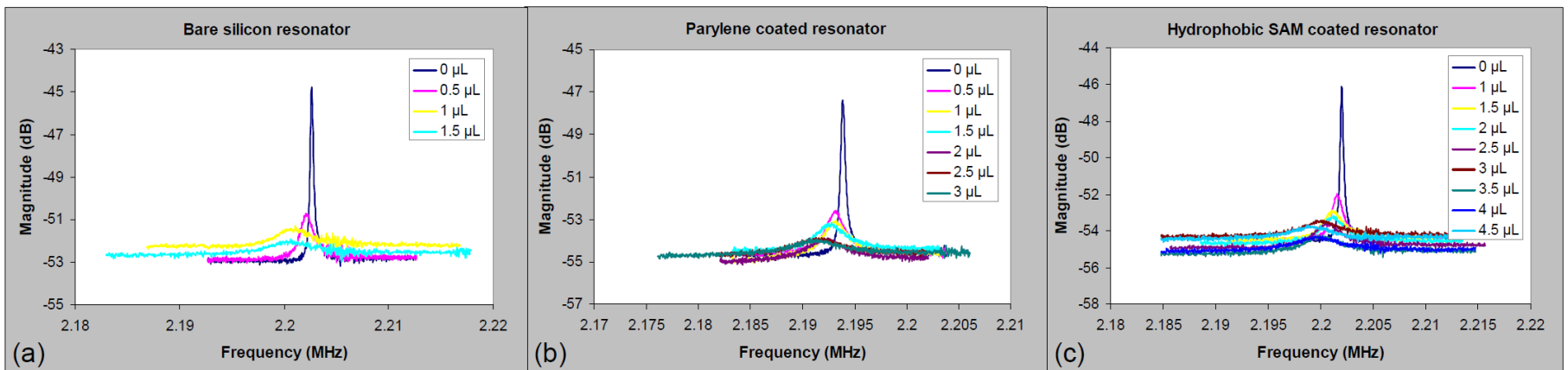

Figure 4: Measured frequency response for a range of water droplet volume spotted in the centre of (a) bare silicon resonator, (b) parylene coated resonator (c) hydrophobic SAM coated resonator.

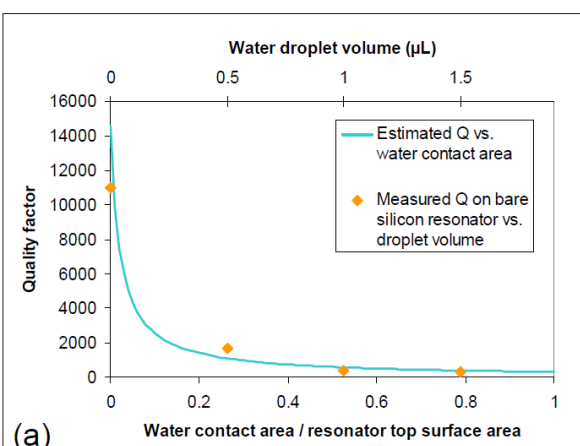

(a)

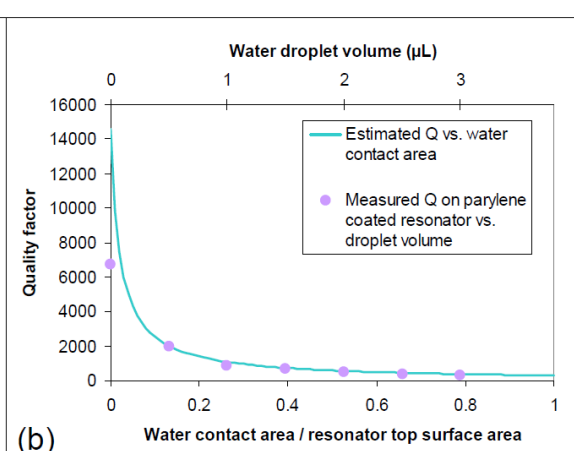

(b)

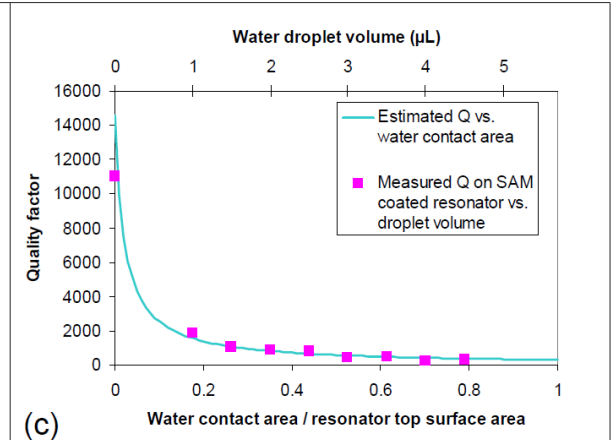

(c)

Figure 5: Comparison between measured Q and the analytical model for (a) bare silicon resonator, (b) parylene coated resonator and (c) hydrophobic SAM coated resonator. The maximum water droplet volume that can be applied to each resonator is assumed to have covered $78 \%$ of the top surface from optical inspection. 
Table 2: Summary of measured resonant frequency and Q factor.

\begin{tabular}{|c|c|c|c|c|c|c|}
\hline \multirow{2}{*}{$\begin{array}{c}\text { Water } \\
\text { droplet size } \\
(\mu \mathrm{L})\end{array}$} & \multicolumn{2}{|c|}{ Bare silicon resonator } & \multicolumn{2}{|c|}{ Parylene coated resonator } & \multicolumn{2}{|c|}{ Hydrophobic SAM coated resonator } \\
\hline & $\begin{array}{c}\text { Resonant frequency } \\
(\mathrm{Hz})\end{array}$ & Quality factor & $\begin{array}{c}\text { Resonant frequency } \\
(\mathrm{Hz})\end{array}$ & Quality factor & $\begin{array}{c}\text { Resonant frequency } \\
(\mathrm{Hz})\end{array}$ & Quality factor \\
\hline 0 & 2202615 & 11013 & 2193855 & 6750 & 2202003 & 11010 \\
\hline 0.5 & 2202090 & 1694 & 2193605 & 1994 & $* * *$ & $* * *$ \\
\hline 1 & 2201060 & 391 & 2193195 & 836 & 2201548 & 1874 \\
\hline 1.5 & 2200640 & 284 & 2192795 & 702 & 2200943 & 1074 \\
\hline 2 & $* * *$ & $* * *$ & 2192390 & 466 & 2201280 & 870 \\
\hline 2.5 & $* * *$ & $* * *$ & 2191880 & 365 & 2200633 & 782 \\
\hline 3 & $* * *$ & $* * *$ & 2190735 & 319 & 2199523 & 441 \\
\hline 3.5 & $* * *$ & $* * *$ & $* * *$ & $* * *$ & 2199823 & 506 \\
\hline 4 & $* * *$ & $* * *$ & $* * *$ & $* * *$ & 2200198 & 275 \\
\hline 4.5 & $* * *$ & $* * *$ & $* * *$ & $* * *$ & 2199973 & 324 \\
\hline
\end{tabular}

The Q factor in air is predicted to be 14574, which is larger than the measured value of 11013 for bare silicon. This is due to the effect of anchor loss not taken into consideration in the model. However, the $\mathrm{Q}$ due to viscous damping can be obtained from total $\mathrm{Q}$ measured in vacuum and in air; $Q_{v i s} \approx\left(1 / Q_{a i r}-1 / Q_{v a c}\right)^{-1}=12957$, which is close to the estimated $Q_{v i s}$. The drop in initial $\mathrm{Q}$ and resonant frequency measured for parylene coated resonator in air indicate that parylene coating itself contributes to more damping and mass loading than air. Similar Q and resonant frequency was measured for hydrophobic SAM coated resonator as compare to bare silicon, showing that hydrophobic SAM coating has very little effect on damping and mass loading in air.

\section{CONCLUSIONS}

The frequency response of three identically designed single crystal silicon resonators with different surface coatings are compared in air with various volumes of water droplets spotted on the resonator surface. One resonator is left bare, the $2^{\text {nd }}$ is coated with $300 \mathrm{~nm}$ of parylene $\mathrm{C}$ and the $3 \mathrm{rd}$ is treated with hydrophobic SAM (perfluorotricholorosilane). Water droplets ranging from 1 to $4.5 \mu \mathrm{L}$ are spotted on the centre of the resonator upper surface, and the resonator response is characterized using differential electrostatic driving and piezoresistive sensing. Hydrophobic SAM coated resonators showed the highest $Q$ for the same water droplet volume applied, indicating that SAM effectively enhanced the performance in a liquid environment due to the reduction in water contact area. However, for similar fluid contact areas, the Qs were similar for all three coatings, indicating that surface coatings may not have an effect on viscous damping for the same effective water contact area. An analytical model describing fluid damping was given to estimate the resonator quality factor for a square extensional mode. The comparison between estimated and measured Q shows that the decreasing trend of the estimated Q is close to the measured Q for all three resonators, and that damping is viscous drag dominated when the ratio of water contact area to resonator top surface area is more than $2 \%$.

\section{ACKNOWLEDGMENT}

The authors would like to thank Dr. T. Adrega and Dr. I. Graz for their assistance in the deposition of hydrophobic SAM and parylene. A. Lin is a Cambridge Commonwealth Scholar, and she gratefully thanks S. Ghosh for the valuable discussions. This work was supported by the US Army Soldier Systems Center.

\section{REFERENCES}

[2] Y. H. Cho, B. M. Kwak, A. P. Pisano, and R. T. Howe, "Viscous

S. D. Senturia, Microsystem Design, 1st ed.: Kluwer Academic Publishers, 2000.

Energy-Dissipation in Laterally Oscillating Planar Microstructures - a Theoretical and Experimental-Study," Micro Electro Mechanical Systems, Proceedings, pp. 93-98, 288, 1993.

[3] F. R. Blom, S. Bouwstra, M. Elwenspoek, and J. H. J. Fluitman, "Dependence of the Quality Factor of Micromachined Silicon Beam Resonators on Pressure and Geometry," Journal of Vacuum Science \& Technology B, vol. 10, pp. 19-26, Jan-Feb 1992.

[4] J. F. Vignola, J. A. Judge, J. Jarzynski, M. Zalalutdinov, B. H. Houston, and J. W. Baldwin, "Effect of viscous loss on mechanical resonators designed for mass detection," Applied Physics Letters, vol. 88, p. 041921, Jan 232006.

[5] S. A. Bhave, D. Gao, R. Maboudian, and R. T. Howe, "Fullydifferential poly-SiC Lame-mode resonator and checkerboard filter," Mems 2005 Miami: Technical Digest, pp. 223-226, 886, 2005.

[6] A. T.-H. Lin, J. E.-Y. Lee, J. Yan, and A. A. Seshia, "Enhanced transduction methods for electrostatically driven MEMS resonators," in Transducers 2009: Technical Digest, Denver, Colorado, USA, 2009.

[7] L. D. Landau and E. M. Lifshitz, Fluid Mechanics, 2nd ed.: Reed Educational and Professional Publishing Ltd, 1987. 